\title{
NKAP functions as an oncogene in Ewing sarcoma cells partly through the AKT signaling pathway
}

\author{
FENG LI ${ }^{1}$, JING-TAO WU ${ }^{2}$, PENG-FEI WANG ${ }^{3}$ and LI-ZHEN QU ${ }^{4}$ \\ ${ }^{1}$ Department of Joint and Sports Medicine, Zaozhuang Municipal Hospital, Zaozhuang, Shandong 277100; \\ ${ }^{2}$ Department of Orthopedics Surgery, Tengnan Hospital of Zaozhuang Mining Group, Jining, Shandong 277000; \\ ${ }^{3}$ Department of Hand, Foot and Microsurgery, Shandong Energy Zaozhuang Mining Group Central Hospital, \\ Zaozhuang, Shandong 277800; ${ }^{4}$ Department of Orthopedics Trauma, Zaozhuang Municipal Hospital, \\ Zaozhuang, Shandong 277100, P.R. China
}

Received October 24, 2018; Accepted July 12, 2019

DOI: $10.3892 /$ etm.2019.7925

\begin{abstract}
NF $-\kappa \mathrm{B}$ activating protein (NKAP) is a highly conserved protein involved in transcriptional repression, immune cell development, maturation, acquisition of functional competency and maintenance of hematopoiesis. In the present study, the function of NKAP in the progress of Ewing sarcoma (ES) was investigated. It was identified that NKAP is highly expressed in ES cells when compared with human mesenchymal stem cells (MSCs). NKAP was knocked-down in human ES cell lines A673 and RD-ES using small interfering (si)RNA transfection. The effectiveness of transfection was then verified using reverse transcription-quantitative PCR and western blot analysis to determine mRNA and protein levels, respectively. The results of the proliferation assays indicated that the knockdown of NKAP inhibited the proliferation and clonogenic abilities of human ES cells. Transwell assays further indicated that cell invasion and migration were significantly inhibited by NKAP knockdown, which may be mediated by downregulation of matrix metalloproteinase (MMP)-9 activity. Gain-of-function analysis also demonstrated the positive role NKAP played in the proliferation, invasion and migration of ES cells. Cell apoptosis was evaluated by flow cytometry, which identified that apoptotic cells were significantly increased when NKAP was silenced. In addition, downregulation of NKAP increased the levels of Bax and cleaved caspase 3, but decreased Bcl2 levels, which suggested that the mitochondrial apoptosis pathway was activated. To explore the action mechanism of NKAP, the status of the AKT signaling pathway in NKAP-silenced A673 and RD-ES cells was investigated. Results indicated that
\end{abstract}

Correspondence to: Dr Li-Zhen Qu, Department of Orthopedics Trauma, Zaozhuang Municipal Hospital, 41 Longtou Road, Zaozhuang, Shandong 277100, P.R. China

E-mail: qlzcsgkkf@163.com

Key words: NF- $\mathrm{kB}$ activating protein, Ewing sarcoma, oncogene, A673, RD-ES, AKT
NKAP knockdown led to decreased phosphorylation of AKT and expression of cyclin D1, a down-stream effector of the AKT signaling pathway, suggesting inactivation of the AKT signaling pathway. In conclusion, the present study revealed that NKAP promoted the proliferation, migration and invasion of ES cells, at least partly, through the AKT signaling pathway, providing new approaches for the therapeutic application of NKAP in ES.

\section{Introduction}

Ewing sarcoma (ES) is a highly aggressive, small, round cell, malignant neoplasm of bone and soft tissue that typically manifests in children and young adults (1). It demonstrates an aggressive clinical behavior with a high-rate of local recurrence and distant metastasis, with $\sim 25 \%$ patients presenting metastases of the bone marrow and lungs at the time of diagnosis, which contributes to the high mortality rate (2). Primary treatment options for ES are traditional methods such as surgical resection, radiotherapy and chemotherapy, curing $\sim 60 \%$ of patients with localized disease (3). By contrast, the overall survival rate for patients with distant metastases treated with traditional methods is $<30 \%(4,5)$. Therefore, the pathogenesis of ES, especially the molecular mechanism involved in metastasis, needs to be elucidated to produce novel treatments.

$\mathrm{NF}-\kappa \mathrm{B}$ activating protein (NKAP) was originally identified as a nuclear localized protein that promotes tumor necrosis factor- and interleukin-1-induced NF- $\kappa \mathrm{B}$ activation (6). Its protein structure contains three domains; Serine and arginine repeats at the $\mathrm{N}$-terminus (RS domain), followed by a basic domain and a C-terminal DUF926 (domain with unknown function) (7). More specifically, NKAP has been identified to interact with RNA-binding proteins through its RS domain in order to regulate RNA splicing and processing, while its basic domain is essential for nuclear localization (7). Functional investigations have found that NKAP is involved in the development, maturation and acquisition of functional competencies of multiple immune cells including $\mathrm{T}$ cells, Invariant Natural Killer T (iNKT) cells and regulatory $\mathrm{T}$ cells (8-12). Furthermore, two other studies have demonstrated 
that NKAP is required for the maintenance and survival of adult hematopoietic stem cells and may serve an important role in mouse neurogenesis $(13,14)$. However, whether NKAP plays a role in tumor progression remains not clearly understood. Li et al (15) reported that SUMOylated NKAP is required for chromosome alignment in mitosis, and that its dysregulation causes chromosomal instability, potentially contributing to tumorigenesis. Liu et al (16) reported that NKAP functions as an oncogene and its expression is induced by $\mathrm{CoCl}_{2}$ treatment in breast cancer via the AKT/mTOR signaling pathway.

In the present study, the role of NKAP in the proliferation, migration and invasion of ES cells was investigated using RNA interference technology and pcDNA transfection. In addition, the potential action mechanisms of NKAP were determined using signaling pathway investigation. The present study identified that NKAP has the potential to serve as a promising therapeutic target for ES.

\section{Materials and methods}

Cell culture and transfection. Human mesenchymal stem cells (MSCs; cat. no. PCS-500-012), and human ES cell lines A673 (cat. no. CRL-1598; authenticated by short tandem repeat profiling), SK-ES-1 (cat. no. HTB-86) and RD-ES (cat. no. HTB-166) were purchased from the American Type Culture Collection. A4573 cells were obtained from the EuroBoNet cell line panel. TC-71 cells (cat. no. ACC-516) were obtained from German Collection of Microorganisms and Cell Cultures. The cells were cultured in Dulbecco's modified Eagle's medium (DMEM; HyClone; GE Healthcare Life Sciences) and supplemented with $10 \%$ FBS (Gibco; Thermo Fisher Scientific, Inc.) under standard culture conditions $\left(37^{\circ} \mathrm{C} ; 95 \%\right.$ humidified air; $5 \% \mathrm{CO}_{2}$ ).

In order to knock down or overexpress the expression of NKAP, ES cells were transfected with either $5 \mathrm{nmol}$ of NKAP-targeted siRNA (siNKAP) or pcDNA-NKAP (Shanghai GeneChem Co., Ltd.) when cells reached $70 \%$

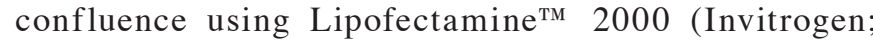
Thermo Fisher Scientific, Inc.) for $5 \mathrm{~h}$ in DMEM. Cells were transferred into DMEM supplemented with 10\% FBS and normally cultured for $24 \mathrm{~h}$ before subsequent experimentation. The sequences of siRNAs were as follows: siNKAP, 5'-GAGAAGAGAGCCCTTGCAT-3'; non-targeting negative control siRNA (siNC; Shanghai GeneChem Co.,Ltd.), 5'-UUCUCCGAACGUGUCACGUTT-3'. siNC was transfected into ES cells as the control of siNKAP.

Reverse transcription-quantitative $P C R$ (RT-qPCR). Following transfection for $24 \mathrm{~h}$, total RNA was isolated from the A673 and RD-ES cells using Ultrapure RNA kit (Beijing CWBio). A total of $1 \mathrm{mg}$ of RNA was transformed into cDNA through a $\mathrm{RT}$ reaction with oligo (dT) primers using HiFiScript cDNA Synthesis kit (Beijing CWBio). The liquid mixture was incubated at $42^{\circ} \mathrm{C}$ for $30-50 \mathrm{~min}$, and then at $85^{\circ} \mathrm{C}$ for $5 \mathrm{~min}$. Then $1 \mathrm{ng}$ of first-strand cDNA was used as a template for qPCR using SYBR Premix Ex Taq II kit (Takara Bio, Inc.; $95^{\circ} \mathrm{C}$ for $10 \mathrm{~min}, 40$ cycles at $95^{\circ} \mathrm{C}$ for $15 \mathrm{sec}$ and at $60^{\circ} \mathrm{C}$ for $\left.1 \mathrm{~min}\right)$. The relative expression of target gene was analyzed using the $2^{-\Delta \Delta \mathrm{Ct}}$ method (17). $\beta$-actin was used as an internal control. Sequences of the primers were as follows:
NKAP forward, 5'-CGGCAGAAGAGATTAAGTGAG-3' and reverse, 5'-CGTTCATACCCCCAGAGGTTTAG-3', and $\beta$-actin forward, 5'-CCCGAGCCGTGTTTCCT-3' and reverse, 5'-GTCCCAGTTGGTGACGATGC-3'.

Western blot analysis. Following transfection for $48 \mathrm{~h}$, MSC, SK-ES-1, TC-71, A4573, A673 and RD-ES cells were collected and lysed in radioimmunoprecipitation assay lysis buffer and protease inhibitors (CWbio). The lysates were centrifuged at $4^{\circ} \mathrm{C}$ and $12,396 \mathrm{x}$ for $30 \mathrm{~min}$, and then protein was quantified using BCA protein assay kit (Sangon Biotech Co., Ltd.). Equal amounts of proteins $(20 \mathrm{mg})$ from each group were loaded into the lanes of a $10 \%$ gel for separation by SDS-PAGE then were electrotransferred onto polyvinylidene fluoride membranes. Non-specific binding was blocked by incubating the membranes with $5 \%$ non-fat milk for $1 \mathrm{~h}$ at room temperature. The membranes were then incubated with primary antibodies overnight at $4^{\circ} \mathrm{C}$, washed with TBST (TBS buffer with $0.1 \%$ Tween 20 ) three times, and incubated with HRP-conjugated secondary antibodies (1:5,000; cat. no. SA00001-15/SA00001-1; ProteinTech Group, Inc.) for $1 \mathrm{~h}$ at room temperature. After being washed with TBST another four times, the protein bands were visualized using the Amersham ECL Prime Western Blotting Detection Reagent (GE Healthcare),, with the gray values being quantified by Image J software v1.8.0 (National Institutes of Health). The primary antibodies against Bax (cat. no. 60267-1-lg; 1:1,000), cleaved caspase-3 (cat. no. 19677-1-AP; 1:10,000), Bcl2 (cat. no. 12789-1-AP; 1:1,000), AKT (cat. no. 60203-2-Ig; 1:5,000), phosphorylated (p)-AKT (cat. no. 66444-1-Ig; 1:2,000), cyclin D1 (cat. no. 60186-1-Ig; 1:10,000) and GAPDH (cat. no. 60004-1-Ig; 1:1,000) were purchased from ProteinTech Group, Inc. The primary antibodies against NKAP (cat. no. ab229096; 1:1,000) and $\alpha$-Tubulin (cat. no. ab7291; 1:10,000) were purchased from Abcam. The primary antibody against Caspase 3 (cat. no. 9662; 1:1,000) was obtained from Cell Signaling Technology. $\alpha$-Tubulin and GAPDH were used as internal controls.

Proliferation assays. For the Cell Counting Kit-8 (CCK-8) assay, A673 and RD-ES cells transfected with siNKAP or pcDNA-NKAP were seeded into a 96-well plate at a density of 3,000 cells per well, each group including three wells. Cells were cultured for 24, 48 and $72 \mathrm{~h}$ then were treated with $10 \mathrm{ml}$ CCK-8 solution (Dojindo Molecular Technologies, Inc.) at $37^{\circ} \mathrm{C}$ for $2 \mathrm{~h}$. Optical density values with $450 \mathrm{~nm}$ were detected using a microplate reader (iMark; Bio-Rad Laboratories, Inc.).

For the colony formation assay, 500 cells transfected with siRNA for $24 \mathrm{~h}$ were seeded in $6-\mathrm{cm}$ petri dishes and cultured in DMEM medium supplemented with $10 \% \mathrm{FBS}$ at $37^{\circ} \mathrm{C}$ for two weeks. The colonies were thereafter dried in the air for $1 \mathrm{~h}$ prior to fixing with $4 \%$ paraformaldehyde at room temperature for $15 \mathrm{~min}$ and staining with $0.1 \%$ crystal violet solution at room temperature for $20 \mathrm{~min}$. The number of colonies was subsequently counted using a light microscope (magnification, $\mathrm{x} 4$ ).

Transwell invasion and migration assays. Transwell chambers (pore size, $8 \mathrm{~mm}$; EMD Millipore) were used to detect the invasion and migration of the ES cells transfected with siNKAP or pcDNA-NKAP. For cell invasion, assay the chambers 
A

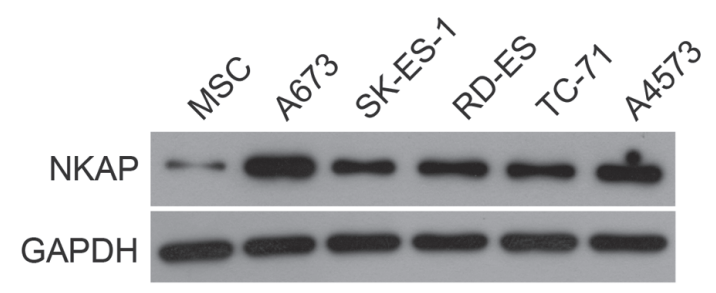

C

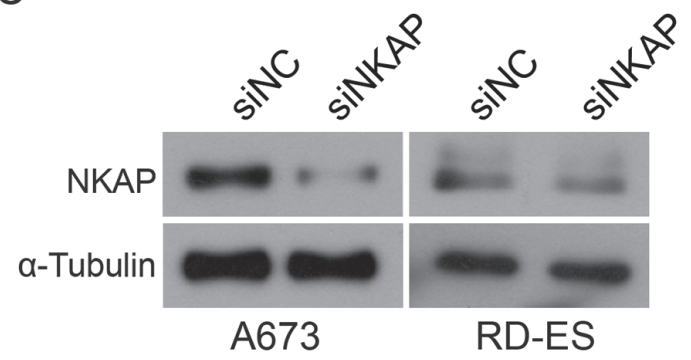

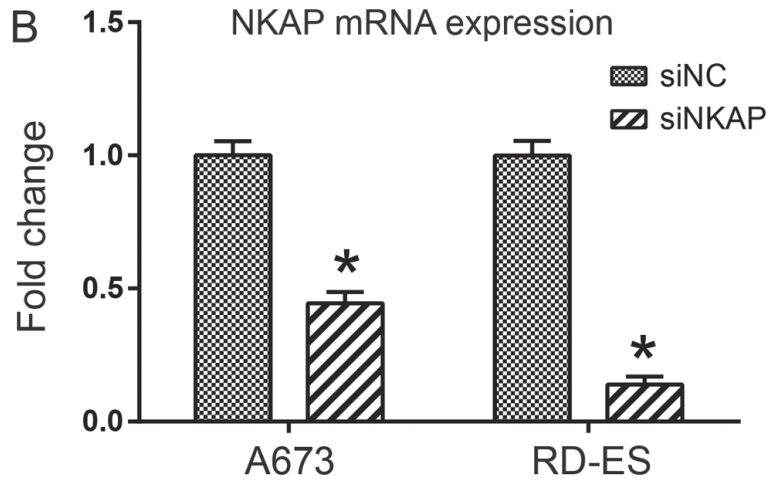

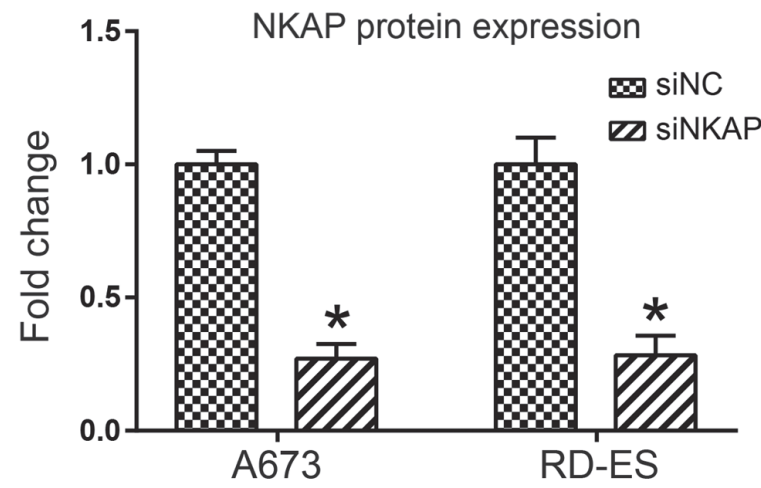

Figure 1. NKAP expression is downregulated in human ES cell lines A673 and RD-ES following siRNA transfection. (A) The expression of NKAP in five different ES cell lines (A673, SK-ES-1, RD-ES, TC-71 and A4573 cells) and MSCs was analyzed using western blot analysis. (B) siRNA targeting NKAP was designed, and introduced into A673 and RD-ES cells with scrambled siRNA being used as the NC. mRNA expression of NKAP was measured using reverse transcription-quantitative PCR and (C) NKAP protein expression was measured using western blot analysis. All experiments were performed in triplicate. ${ }^{*} \mathrm{P}<0.05$ vs. siNC. NKAP, NF- $\kappa \mathrm{B}$ activating protein; ES, Ewing sarcoma; siRNA or si, small-interfering RNA; siNC, negative control.

were pre-coated with Matrigel (BD Biosciences) at $4^{\circ} \mathrm{C}$ for 30 min. Following transfection, A673 or RD-ES cells were seeded in the upper chambers at a density of $1 \times 10^{5}$ in $200 \mu 1$ of serum-free DMEM. The lower chambers were then filled with $500 \mu \mathrm{l}$ of DMEM with $20 \%$ FBS as the chemoattractant. Following $24 \mathrm{~h}$ of incubation at $37^{\circ} \mathrm{C}$, the non-invaded cells on the upper Transwell membrane were removed by scraping, while the invaded cells were fixed with $4 \%$ paraformaldehyde at room temperature for $30 \mathrm{~min}$ and stained with $0.1 \%$ crystal violet at room temperature for $20 \mathrm{~min}$. The stained cells were photographed using a light microscope at magnification $\mathrm{x} 100$ and counted in three random view fields.

The cell migration experiment followed the aforementioned protocol however no Matrigel was used in the Transwell chambers.

Gelatin zymography. The influence of NKAP knockdown on the proteolytic activities of MMP-9 was detected using gelatin zymography. First, the A673 and RD-ES cells were transfected with siNC or siNKAP for $24 \mathrm{~h}$. The cells were then harvested and seeded in a 6 -cm petri dish $\left(1 \times 10^{5}\right.$ cells/dish) with DMEM with $10 \% \mathrm{FBS}$. Following culture for $24 \mathrm{~h}$ at $37^{\circ} \mathrm{C}$, the supernatants were collected and centrifuged at $4^{\circ} \mathrm{C}$ and $2,066 \mathrm{x} \mathrm{g}$ for $5 \mathrm{~min}$ to remove cell debris. Protein concentrations were quantified with the BCA protein assay kit (Sangon Biotech Co., Ltd.). A total of $20 \mu \mathrm{g}$ of proteins from each sample were then loaded into a $10 \%$ gel with $1 \mathrm{mg} / \mathrm{ml}$ gelatin A (Sigma-Aldrich; Merck KGaA) and separated by SDS/PAGE for $1.5 \mathrm{~h}$. Finally, the gels were incubated with $0.1 \%$ Coomassie Brilliant Blue at room temperature for $3 \mathrm{~h}$ then destained with $45 \%$ methanol and $10 \%(\mathrm{v} / \mathrm{v})$ acetic acid until clear bands suggestive of gelatin digestion were present. Bands were photographed using a gel imager (Bio-Rad Laboratories, Inc.) and analyzed with Quantity One software v4.5 (Bio-Rad Laboratories, Inc.).

Flow cytometry detection for apoptosis. After being transfected with siNC or siNKAP for $48 \mathrm{~h}$, the apoptosis of ES cells was evaluated using flow cytometry. A total of $1 \times 10^{6} \mathrm{ES}$ cells were centrifuged at $4^{\circ} \mathrm{C}$ and $1,033 \times \mathrm{g}$ for $5 \mathrm{~min}$ and resuspended in $1 \mathrm{ml}$ PBS. A total of $5 \mu \mathrm{l}$ Annexin V $(1 \mu \mathrm{g} / \mathrm{ml}$; Aposcreen annexin v-biot; Beckman Coulter, Inc.) was then added and incubated at room temperature for $15 \mathrm{~min}$. Subsequently, propidium iodide (PI; $1 \mu \mathrm{g} / \mathrm{ml}$; Beckman Coulter, Inc.) was added for $5 \mathrm{~min}$ at room temperature. All staining incubation steps were performed in dark. The apoptosis rate was analyzed by a flow cytometer and calculated using BD FACSDiva software v4.1 (BD Biosciences).

Statistical analysis. All experiments were repeated three times with the data expressed as mean \pm standard deviation. Comparisons between different groups were performed using Student's t-test for two groups or one-way analysis of variance followed by a Tukey's post-hoc test for multiple groups. Statistical analysis was performed using GraphPad Prism 7.0 (GraphPad Software, Inc.). P $<0.05$ was considered to indicate statistical significance. 
A The proliferation of $A 673$ cells

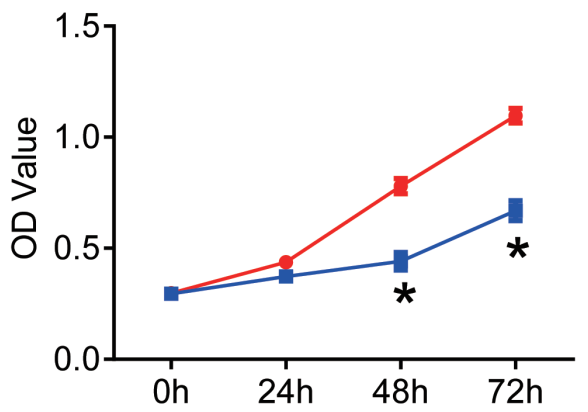

B The proliferation of RD-ES cells

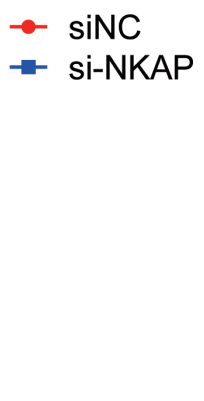

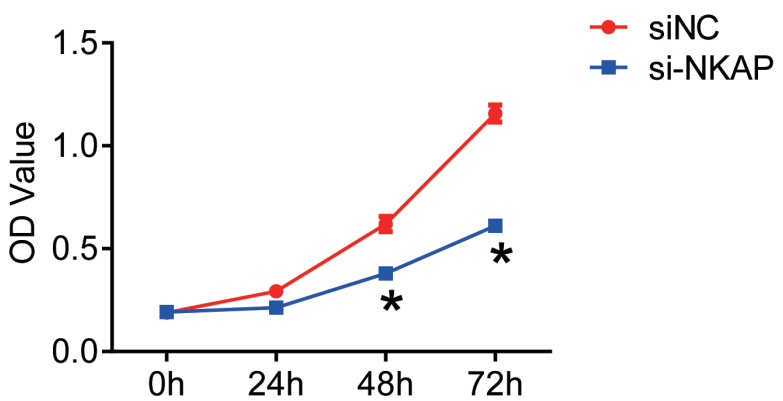

C
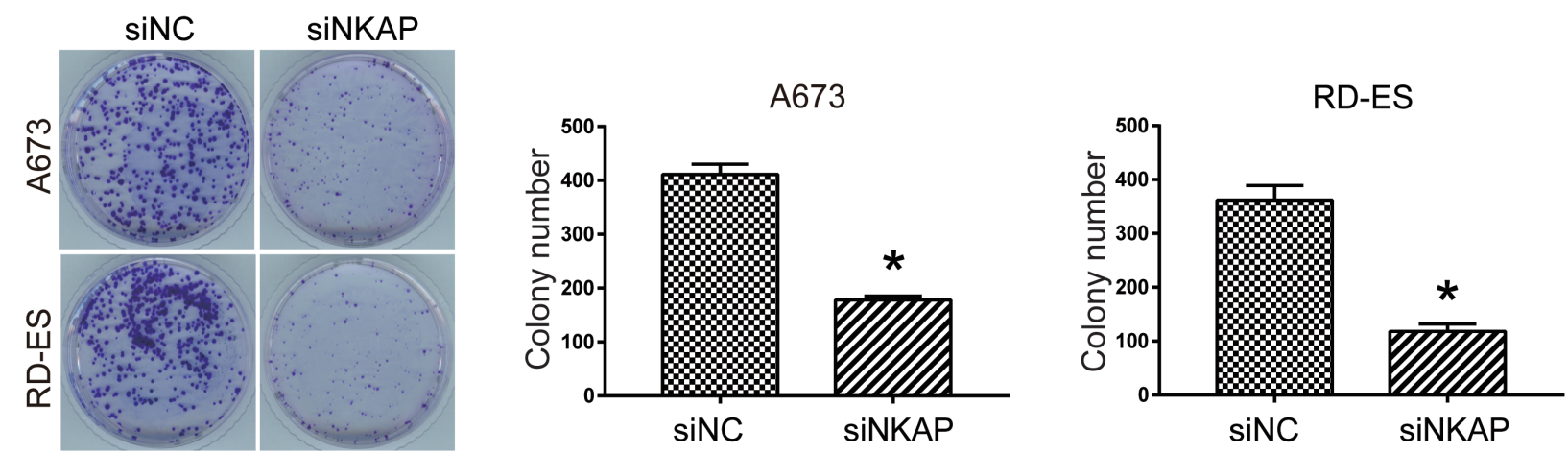

Figure 2. Downregulation of NKAP induces growth inhibition in human ES cells. ES cells were transfected with siNC or siNKAP. The cell viability of (A) A673 and (B) RD-ES cells were measured using the Cell Counting Kit-8 assay. (C) Representative images and (D) quantification of a colony formation assay in A673 and RD-ES cells. All experiments were performed in triplicate. "P<0.05 vs. NC. NKAP, NF- $\mathrm{kB}$ activating protein; ES, Ewing sarcoma; siRNA, small-interfering RNA; siNC, negative control; OD, optical density.

\section{Results}

Downregulation of NKAP induces growth inhibition of ES cells. To investigate the biological functions of NKAP in ES, the expression of NKAP in five different ES cell lines (A673, SK-ES-1, RD-ES, TC-71 and A4573 cells) and MSCs was analyzed using western blot analysis. Results indicated that NKAP was markedly upregulated in ES cell lines when compared with MSCs (Fig. 1A). Due to the high expression of NKAP in A673 and RD-ES cell lines, NKAP was knocked down in human A673 and RD-ES cell lines via siRNA transfection to investigate the biological functions of NKAP. The interference effects of siNKAP were evaluated using RT-qPCR and western blot assays. As demonstrated in Fig. 1B, NKAP mRNA expression was significantly decreased in A673 and RD-ES cells transfected with siNKAP compared with siNC transfection $(\mathrm{P}<0.05)$. Consistent with the mRNA level, NKAP protein expression was also downregulated when NKAP was silenced ( $\mathrm{P}<0.05$; Fig. 1C). Effects of downregulation of NKAP on cell proliferation were investigated using CCK-8 and colony formation assays. Findings determined that siNKAP-transfected A673 and RD-ES cells exhibited significantly decreased cell proliferation and colony formation efficiency when compared with cells transfected with siNC (P<0.05; Fig. 2).

Downregulation of NKAP inhibits the invasion and migration of ES cells by regulating MMP-9 activity. Invasion and migration are important characteristics of cancer cells, frequently initiating tumor metastasis in vivo (18). Therefore, it was investigated whether NKAP was involved in the invasion and migration of ES cells. As shown by the Transwell assays, the number of invasive and migratory A673 cells were significantly decreased when NKAP was knocked down when compared with the $\mathrm{NC}$ group $(\mathrm{P}<0.05$; Fig. $3 \mathrm{~A}$ and $\mathrm{B})$. Similar results were observed in the RD-ES cells $(\mathrm{P}<0.05$; Fig. $3 \mathrm{C}$ and D). In order to determine whether NKAP knockdown induced a decrease in the activity of secreted MMP-9, which might explain the reduced invasion and migration, the medium of ES cells was analyzed for MMP-9 activity by gelatin zymography. As shown in Fig. 3E and F, the degradation of secreted MMP-9 was significantly decreased in both A673 and RD-ES cells following NKAP silencing compared with the control $(\mathrm{P}<0.05)$.

Overexpression of NKAP promotes the viability, invasion and migration of ES cells. Gain-of-function analysis in ES cells was used to investigate the function of NKAP more comprehensively. Overexpression efficiencies of NKAP in A673 and RD-ES cells were confirmed as NKAP expression in NKAP overexpressing vector-transfected cells was significantly greater compared with the control group ( $\mathrm{P}<0.05$; Fig. 4A). A673 and RD-ES cell viability significantly increased when NKAP was overexpressed following pcDNA transfection compared with the control at $72 \mathrm{~h}(\mathrm{P}<0.05$; Fig. 4B and $\mathrm{C})$. In addition, Transwell assays determined that NKAP overexpression had 

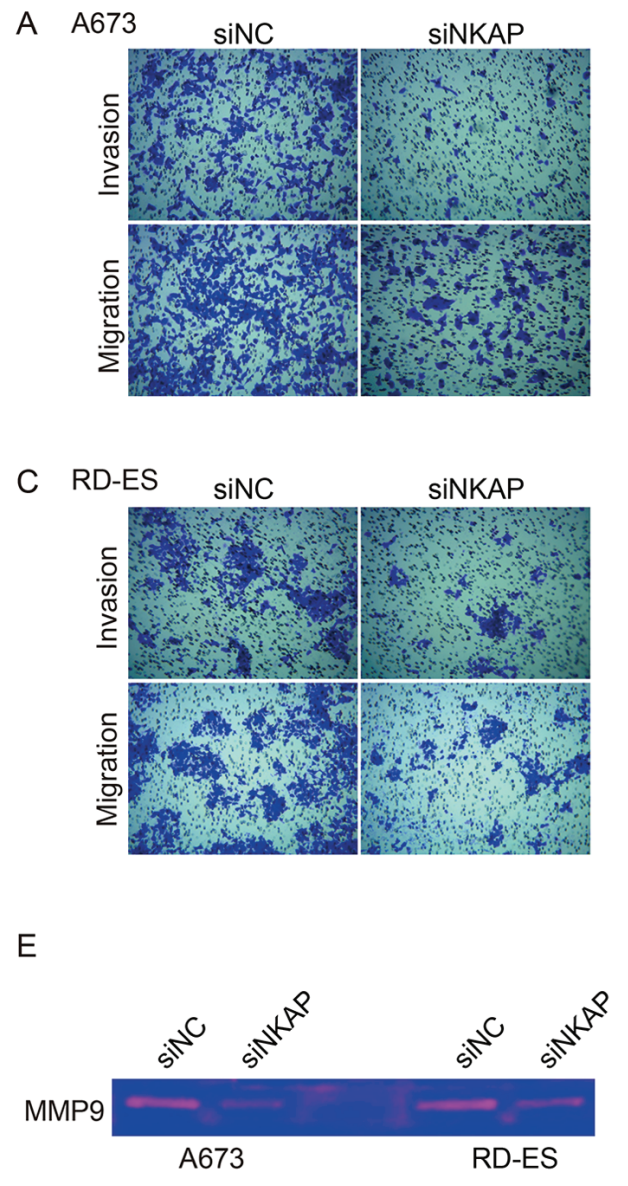

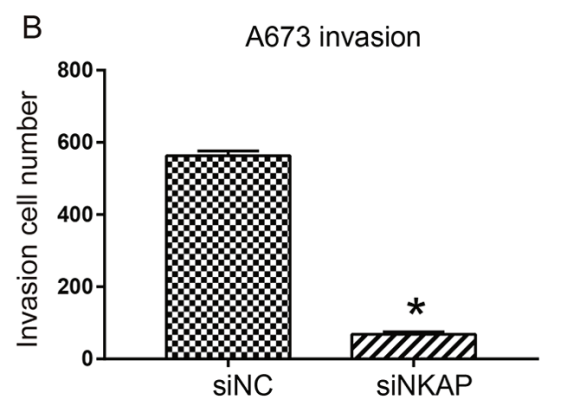

D
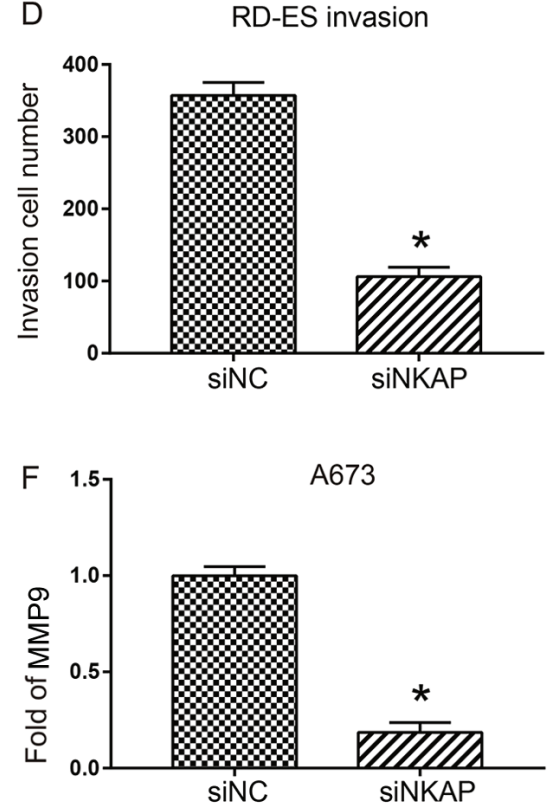

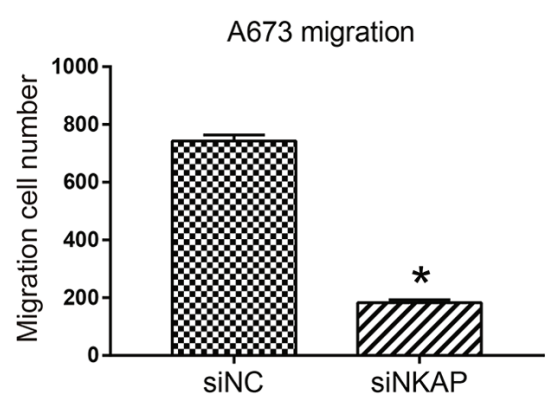

RE-DS migration
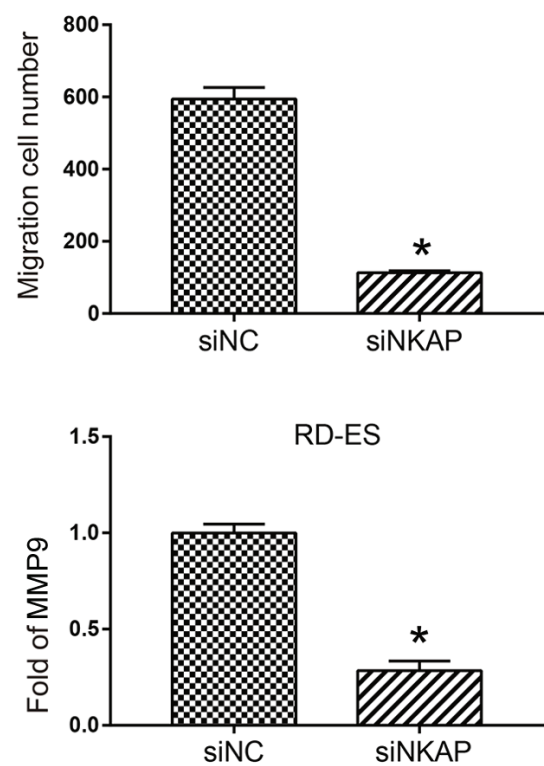

Figure 3. Downregulation of NKAP inhibits the invasion and migration of ES cells by regulating MMP-9 activity. ES cells were transfected with siNC or siNKAP. (A) Representative images and (B) quantification of Transwell invasion and migration in A673 cells. (C) Representative images and (D) quantification of Transwell invasion and migration in RD-ES cells (magnification, $x 100$ ). (E) Representative images and (F) quantification of proteolytic activity of secreted MMP-9 in A673 and RD-ES cells. All experiments were performed in triplicate. "P<0.05 vs. siNC. NKAP, NF-kB activating protein; ES, Ewing sarcoma; MMP, matrix metalloproteinase; siRNA or si, small-interfering RNA; siNC, negative control.

a positive role in significantly increasing the invasion and migration of ES cell lines compared with the control $(\mathrm{P}<0.05$; Fig. 4D-G). Taken together, theses results demonstrated that overexpression of NKAP increased the viability, invasion and migration of ES cells.

Downregulation of NKAP promotes ES cell apoptosis and activates the mitochondrial apoptosis pathway. In order to investigate whether cell apoptosis contributed to the inhibitory effect of NKAP knockdown on ES cells, flow cytometry analysis was performed. As demonstrated in Fig. 5, the apoptosis percentage $\left(Q_{2}+Q_{4}\right)$ significantly increased when NKAP was silenced in A673 and RD-ES cells compared with the control $(\mathrm{P}<0.05)$. Whether the pro-apoptosis effect of NKAP knockdown was mediated by the mitochondrial apoptosis pathway was investigated next. Western blot analysis suggested that NKAP knockdown led to a significant increase in the expression of pro-apoptosis factors, including Bax and cleaved caspase 3, and a decrease of anti-apoptotic member Bcl 2 compared with the control group $(\mathrm{P}<0.05$; Fig. $6 \mathrm{~A}$ and $\mathrm{B})$. Taken together, NKAP knockdown resulted in the activation of the mitochondrial apoptosis pathway, increasing the apoptosis of ES cells.
Downregulation of NKAP inhibits the activation of the AKT signaling pathway. Finally, the mechanism of action of NKAP in ES cells was investigated by focusing on the status of the AKT signaling pathway as it has an important role in tumorigenesis and progression, being involved in cell proliferation, apoptosis and metastasis $(19,20)$. Western blot analysis demonstrated that silencing of NKAP downregulated both the phosphorylation level of AKT and the expression of its down-stream effector cyclin D1 in both A673 and RD-ES cells compared with the control $(\mathrm{P}<0.05$; Fig. $6 \mathrm{C}$ and $\mathrm{D})$. These findings suggested NKAP knockdown led to inactivation of the AKT signaling pathway.

\section{Discussion}

ES is the second-most frequent, primary malignant bone tumor in children and adolescents (1). Though great improvements have been achieved in ES therapy, the prognosis for patients with metastasized ES still remains poor (4). Scientists have thus been committed to identifying effective prognostic markers or therapeutic targets. The present study hypothesized that NKAP has the potential to serve an important role in 
A

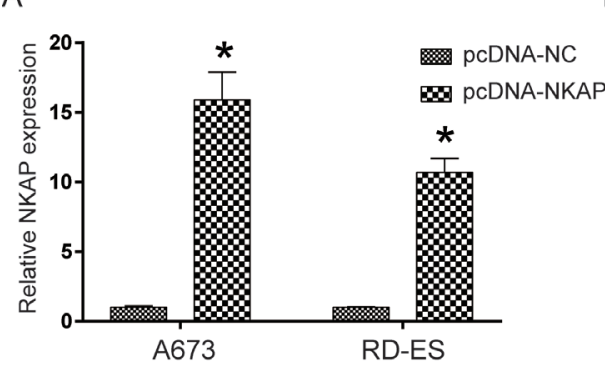

B
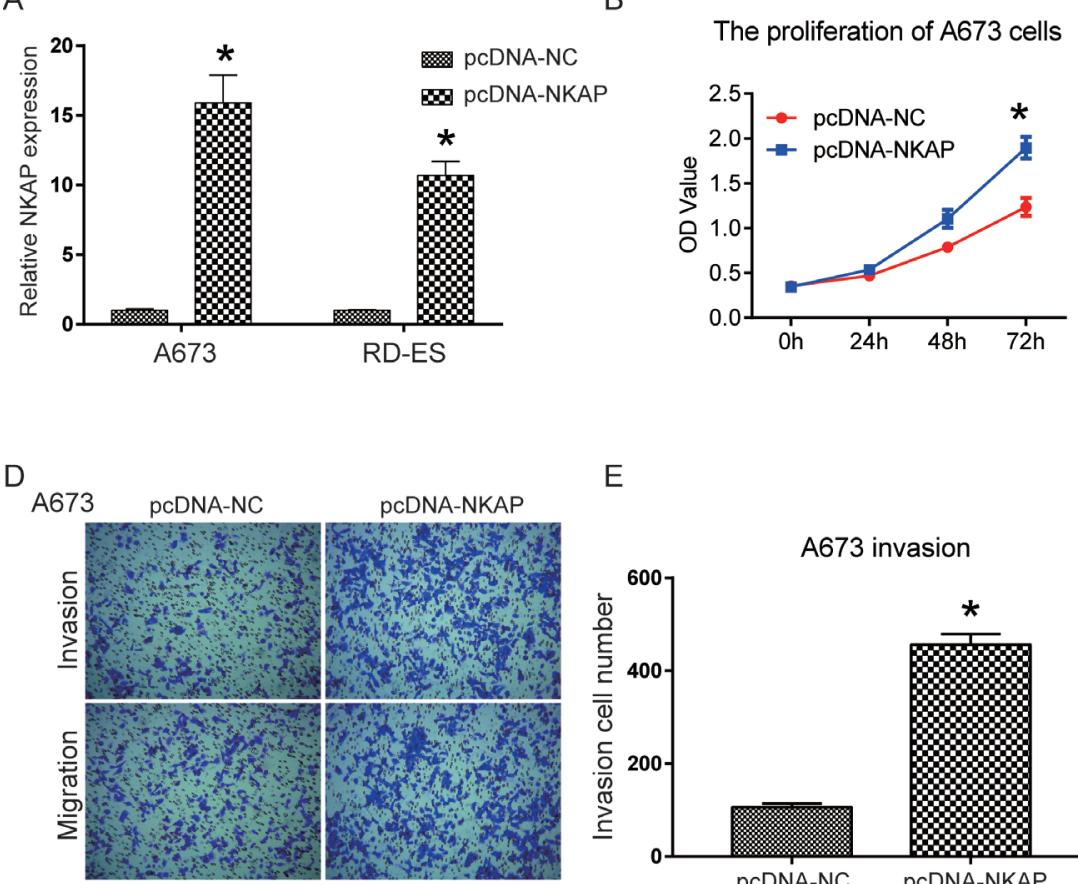

$\mathrm{F}$

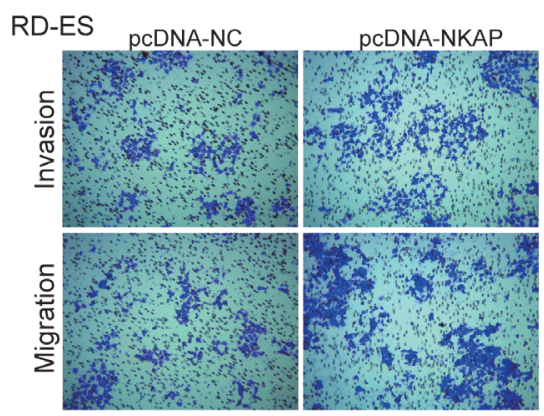

$E$

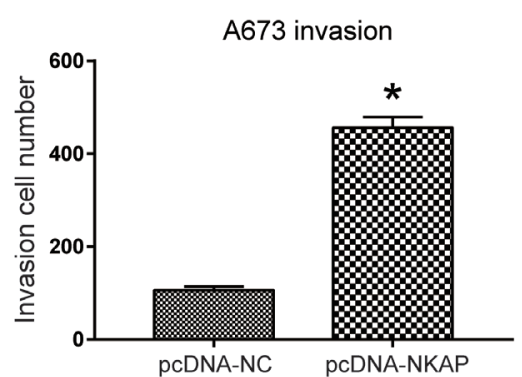

G

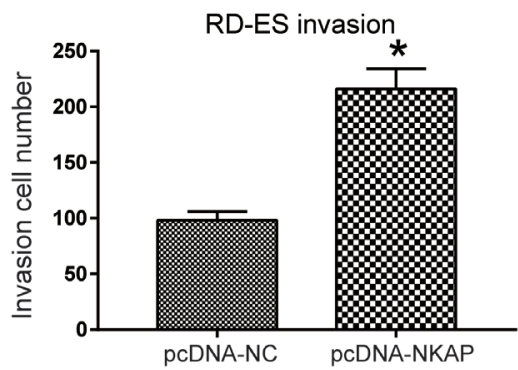

C

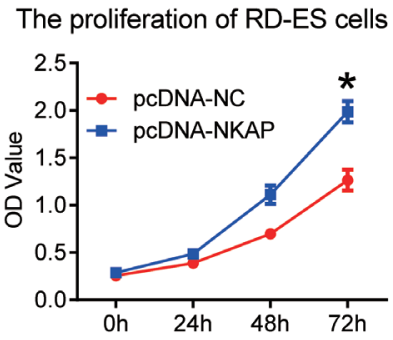

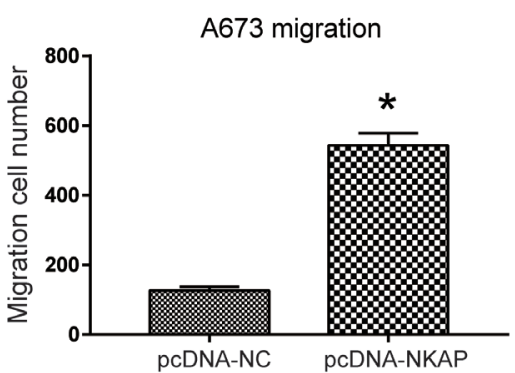

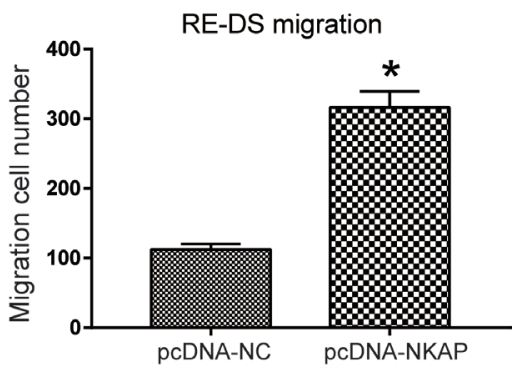

Figure 4. Overexpression of NKAP promotes the proliferation, invasion and migration of ES cells. ES cells were transfected with pcDNA-NC or pcDNA-NKAP. (A) NKAP mRNA expression in A673 and RD-ES cells was examined using reverse transcription-quantitative PCR. (B) Proliferation of A673 and (C) RD-ES cells was determined by the Cell Counting Kit-8 assay. (D) Representative images and (E) quantification of Transwell invasion and migration in A673 cells. (F) Representative images (magnification, x100) and (G) quantification of Transwell invasion and migration in RD-ES cells. *P<0.05 vs. pcDNA-NC. NKAP, $\mathrm{NF}-\kappa \mathrm{B}$ activating protein; ES, Ewing sarcoma; NC, negative control.

tumor related functions. Previous studies have demonstrated that NKAP is a highly conserved protein with various roles in transcription repression, immune development and maturation, the maintenance and survival of adult hematopoietic stem cells, chromosome alignment in mitosis, and RNA splicing and processing $(7,11,14,15)$. Furthermore, NKAP deficiency has been identified in soft tissue sarcomas as well as several other types of human cancer (15). However, the functions and mechanisms of action of NKAP in tumorigenesis and progression need to be further elucidated. Therefore, the present study investigated whether NKAP functions as an important regulator in the proliferation, migration and invasion of ES cells.

In order to determine this, NKAP was knocked down in ES cells lines A673 and RD-ES. The effects of NKAP silencing on the tumor-related phenotypes of ES cells were investigated. Results determined that NKAP knockdown induced the inhibition of both cell proliferation and clonogenic abilities in ES cells, whereas NKAP overexpression promoted cell viability. Flow cytometry indicated that cell apoptosis was promoted in ES cells following NKAP silencing. Previous studies demonstrated that NKAP depletion inhibits the proliferation of iNKT in mice, leading to severe reductions in thymic and peripheral iNKT cell numbers (8), and decreased proliferation and increased apoptosis of hematopoietic stem cells (14). This suggests that NKAP functions as an important regulator in cell proliferation. The present study identified that NKAP knockdown inhibits migration and invasion in ES cell lines, most likely mediated by downregulation of MMP-9 secretion. The MMP family has an essential role in regulating cell mobility by degrading the extracellular matrix to promote tumor metastasis $(21,22)$. Taken together, the present findings identified NKAP as a potential novel therapeutic target for tumor metastasis.

Emerging evidence has identified that NKAP is involved in the transcription repression of Notch (9). In general, NKAP binds CBF1-interacting corepressor and recruits histone deacetlylase in T-cell development, also inducing the activation of the NF- $\kappa \mathrm{B}$ signaling pathway (13). The present study identified that NKAP knockdown led to the activation of the mitochondrial apoptosis pathway as evidenced by the increased levels of Bax and cleaved caspase-3 as well as 
A

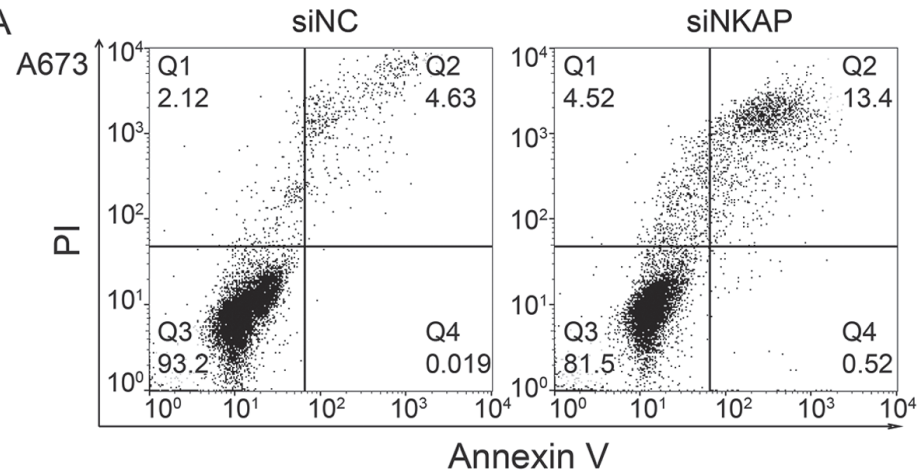

C

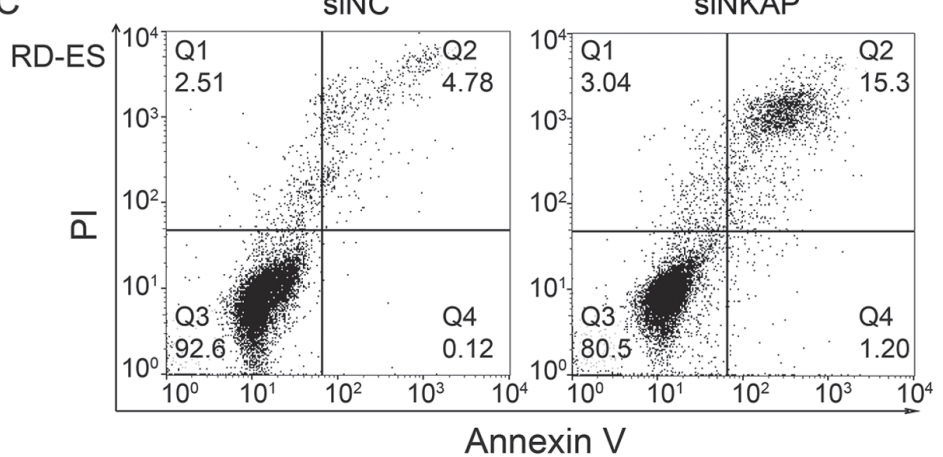

B

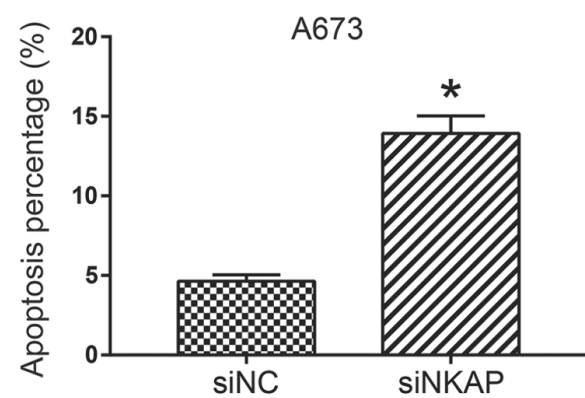

D

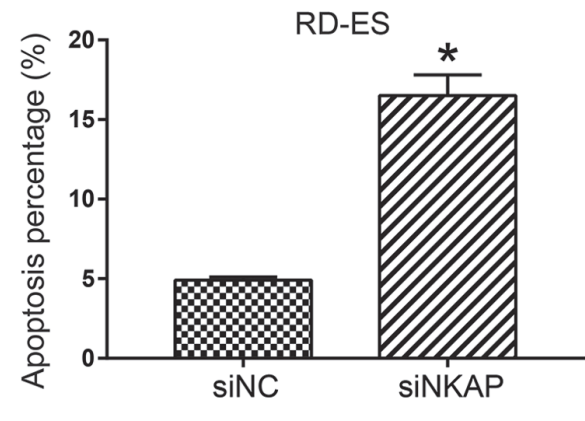

Figure 5. Downregulation of NKAP promotes cell apoptosis in ES cells. (A) Flow cytometry images and (B) quantification of apoptosis were detected using Annexin V/PI staining in A673 cells. (C) Flow cytometry images and (D) quantification of apoptosis were detected using Annexin V/PI staining in RD-ES cells. All experiments were performed in triplicate. " $\mathrm{P}<0.05$ vs. siNC. NKAP, NF- $\kappa \mathrm{B}$ activating protein; ES, Ewing sarcoma; PI, propidium iodide; siRNA or si, small-interfering RNA; siNC, negative control.

A

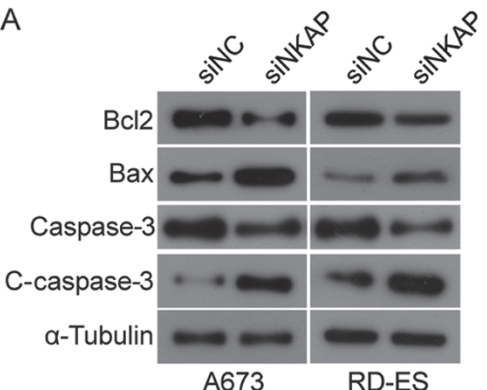

C

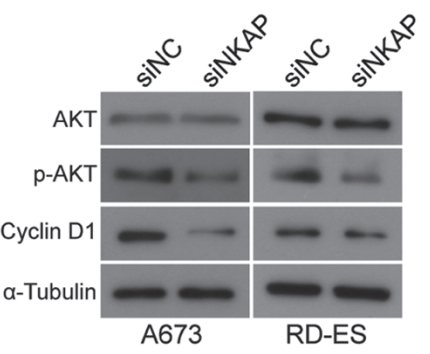

B
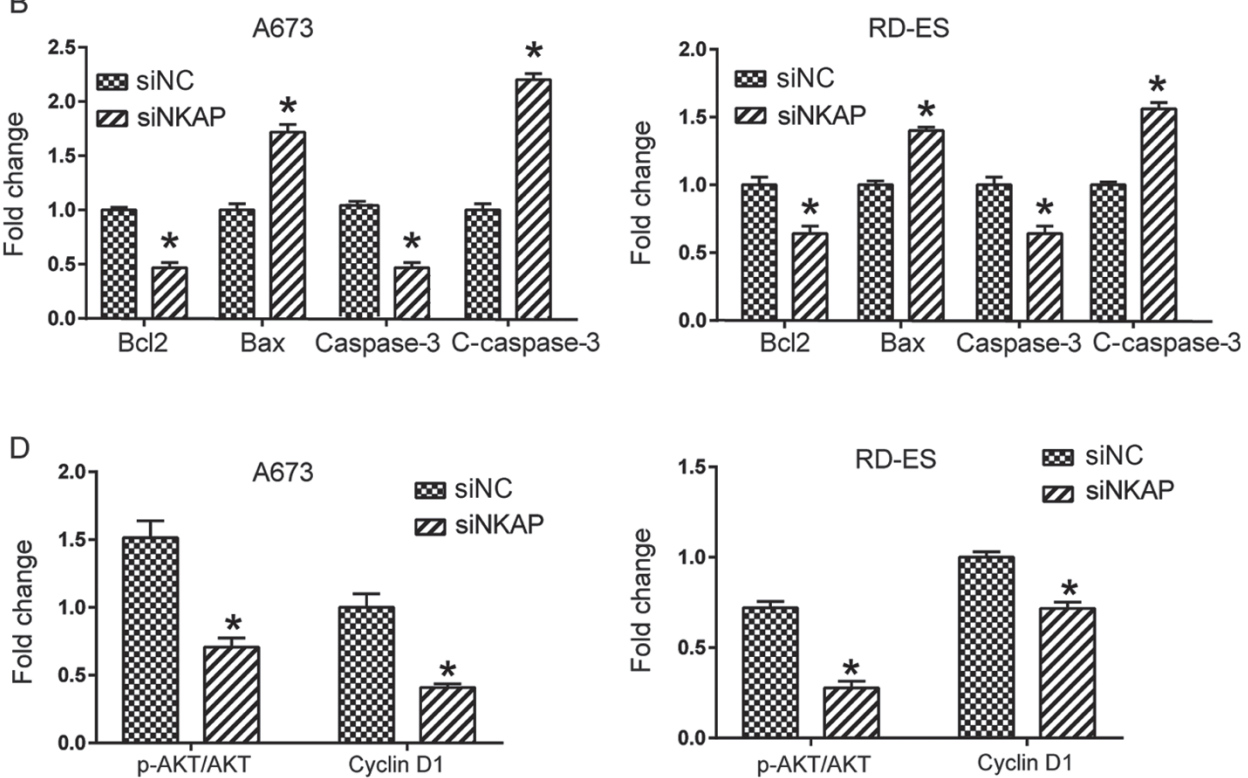

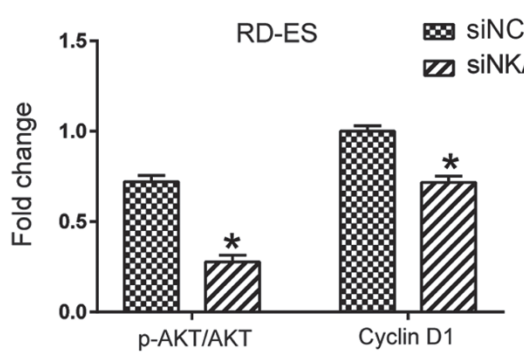

Figure 6. Downregulation of NKAP leads to the activation of the mitochondrial apoptosis pathway and inactivation of the AKT signaling pathway. (A) Representative blots and (B) quantification of mitochondrial apoptosis pathway components Bcl2, Bax, caspase 3 and C-caspase 3 following silencing of NKAP in human A673 and RD-ES cells. (C) Representative blots and (D) quantification of AKT signaling pathway components AKT, p-AKT and cyclin D1 were detected using western blot in human A673 and RD-ES cells following silencing of NKAP. All experiments were performed in triplicate. "P<0.05 vs. siNC. NKAP, NF- $\mathrm{kB}$ activating protein; p, phosphorylated; siRNA or si, small-interfering RNA; siNC, negative control; C, cleaved.

decreased levels of Bcl2 detected by western blot analysis. Bax is an established key apoptosis initiation protein that promotes the permeability of the mitochondrial outer membrane and triggers the release of cytochrome $\mathrm{C}$ to the cytoplasm $(23,24)$. In turn, cytochrome $\mathrm{C}$ induces cell apoptosis via activation of the caspase cascade $(23,24)$. In addition, $\mathrm{Bcl} 2$ functions as 
an anti-apoptotic protein by antagonizing Bax $(25,26)$. The present study determined that the AKT signaling pathway was inactivated in NKAP-silenced ES cells as evidenced by the decreased levels of p-AKT and cyclin D1. The AKT signaling pathway is a key pathway in the regulation of multiple biological processes such as promoting the proliferation, survival and migration of tumors (27). When the AKT pathway is activated, the phosphorylation level of AKT is elevated, which promotes the expression of a number of its downstream effectors important in cellular growth such as cyclin D1 (28). The activation of the Akt/mTOR signaling pathway results in enhanced cell proliferation, finally leading to tumorigenesis (27).

The present study has some limitations. The involvement of the mitochondrial apoptosis pathway should be further confirmed by assessing additional specific markers (including JC-1), following knockdown of NKAP. In addition, no rescue experiments were performed to verify that the oncogenic effects of NKAP on ES cells were indeed mediated by AKT. These areas of focus should be investigated further in the future studies.

In conclusion, the present study revealed that NKAP functions as an oncogenic gene in the progression of ES partly via the mitochondrial apoptosis and AKT pathways. The findings suggested that NKAP could be a novel therapeutic target for ES.

\section{Acknowledgements}

Not applicable.

\section{Funding}

No funding was received.

\section{Availability of data and materials}

The datasets used and/or analyzed during the present study are available from the corresponding author on reasonable request.

\section{Authors' contributions}

FL and LQ designed the study and wrote the manuscript. FL, JW and PW performed the experiments and statistical analysis. All authors read and approved the final manuscript.

\section{Ethics approval and consent to participate}

Not applicable.

\section{Patient consent for publication}

Not applicable.

\section{Competing interests}

The authors declare that they have no competing interests.

\section{References}

1. Esiashvili N, Goodman M and Marcus RB Jr.: Changes in incidence and survival of Ewing sarcoma patients over the past 3 decades-surveillance epidemiology and end results data. J Pediatr Hematol Oncol 30: 425-430, 2008.
2. Galyfos G, Karantzikos GA, Kavouras N, Sianou A, Palogos K and Filis K: Extraosseous ewing sarcoma: Diagnosis, prognosis and optimal management. Indian J Surg 78: 49-53, 2016.

3. Max D, Kuehnoel CD, Burdach S, Niu L, Staege MS and Foell JL: Indoleamine-2,3-dioxygenase in an immunotherapy model for ewing sarcoma. Anticancer Res 34: 6431-6441, 2014.

4. Ladenstein R, Poetschger U, Le Deley MC, Whelan J, Paulussen M, Oberlin O, van den Berg H, Dirksen U, Hjorth L, Michon J, et al: Primary Disseminated Multifocal Ewing Sarcoma: Results of the Euro-EWING 99 Trial. J Clin Oncol 28: 3284-3291, 2010.

5. Sand LG, Szuhai K and Hogendoorn PC: Sequencing Overview of ewing sarcoma: A journey across genomic, epigenomic and transcriptomic landscapes. Int J Mol Sci 16: 16176-16215, 2015.

6. Chen D, Li Z, Yang Q, Zhang J, Zhai Z and Shu HB: Identification of a nuclear protein that promotes NF-kappaB activation. Biochem Biophys Res Commun 310: 720-724, 2003.

7. Burgute BD, Peche VS, Steckelberg AL, Glöckner G, Gaßen B, Gehring NH and Noegel AA: NKAP is a novel RS-related protein that interacts with RNA and RNA binding proteins. Nucleic Acids Res 42: 3177-3193, 2014.

8. Thapa P, Chen MW, McWilliams DC, Belmonte P, Constans M, Sant'Angelo DB and Shapiro VS: NKAP Regulates Invariant NKT Cell Proliferation and Differentiation into ROR-gammat-Expressing NKT17 Cells. J Immunol 196: 4987-4998, 2016.

9. Pajerowski AG, Nguyen C, Aghajanian H, Shapiro MJ and Shapiro VS: NKAP is a transcriptional repressor of notch signaling and is required for $\mathrm{T}$ cell development. Immunity 30 : 696-707, 2009.

10. Thapa P, Das J, McWilliams D, Shapiro M, Sundsbak R, Nelson-Holte M, Tangen S, Anderson J, Desiderio S, Hiebert S, et al: The transcriptional repressor NKAP is required for the development of iNKT cells. Nat Commun 4: 1582, 2013.

11. Hsu FC, Pajerowski AG, Nelson-Holte M, Sundsbak R and Shapiro VS: NKAP is required for T cell maturation and acquisition of functional competency. J Exp Med 208: 1291-1304, 2011.

12. Dash B, Shapiro MJ, Chung JY, Romero Arocha S and Shapiro VS: Treg-specific deletion of NKAP results in severe, systemic autoimmunity due to peripheral loss of Tregs. J Autoimmun 89: 139-148, 2018.

13. Worlitzer MM and Schwamborn JC: The Notch co-repressor protein NKAP is highly expressed in adult mouse subventricular zone neural progenitor cells. Neuroscience 266: 138-149, 2014.

14. Pajerowski AG, Shapiro MJ, Gwin K, Sundsbak R Nelson-Holte M, Medina K and Shapiro VS: Adult hematopoietic stem cells require NKAP for maintenance and survival. Blood 116: 2684-2693, 2010.

15. Li T, Chen L, Cheng J, Dai J, Huang Y, Zhang J, Liu Z, Li A, Li N, Wang H, et al: SUMOylated NKAP is essential for chromosome alignment by anchoring CENP-E to kinetochores. Nat Commun 7: 12969, 2016.

16. Liu J, Wang H, Yin Y, Li Q and Zhang M: NKAP functions as an oncogene and its expression is induced by $\mathrm{CoCl} 2$ treatment in breast cancer via AKT/mTOR signaling pathway. Cancer Manag Res 10: 5091-5100, 2018.

17. Livak KJ and Schmittgen TD: Analysis of relative gene expression data using real-time quantitative PCR and the 2 (-Delta Delta C (T)) method. Methods 25: 402-408, 2001.

18. Kalluri R and Weinberg RA: The basics of epithelial-mesenchymal transition. J Clin Invest 119: 1420-1428, 2009.

19. Franke TF, Hornik CP, Segev L, Shostak GA and Sugimoto C: PI3K/Akt and apoptosis: Size matters. Oncogene 22: 8983-8998, 2003.

20. Lassen A, Atefi M, Robert L, Wong DJ, Cerniglia M, Comin-Anduix B and Ribas A: Effects of AKT inhibitor therapy in response and resistance to BRAF inhibition in melanoma. Mol Cancer 13: 83, 2014.

21. Choi BD, Jeong SJ, Wang G, Park JJ, Lim DS, Kim BH, Cho YI, Kim CS and Jeong MJ: Secretory leukocyte protease inhibitor is associated with MMP-2 and MMP-9 to promote migration and invasion in SNU638 gastric cancer cells. Int J Mol Med 28: 527-534, 2011.

22. Zhang X, Min J, Wang Y, Li Y, Li H, Liu Q, Liang X, Mu P and Li H: RABEX-5 plays an oncogenic role in breast cancer by activating MMP-9 pathway. J Exp Clin Cancer Res 32: 52, 2013.

23. Gui D, Guo Y, Wang F, Liu W, Chen J, Chen Y, Huang J and Wang N: Astragaloside IV, a novel antioxidant, prevents glucose-induced podocyte apoptosis in vitro and in vivo. PLoS One 7: e39824, 2012. 
24. Martinou JC and Youle RJ: Mitochondria in apoptosis: Bcl-2 family members and mitochondrial dynamics. Dev Cell 21: 92 , 2011.

25. Yang TQ, Lu XJ, Wu TF, Ding DD, Zhao ZH, Chen GL, Xie XS, Li B, Wei YX, Guo LC, et al: MicroRNA-16 inhibits glioma cell growth and invasion through suppression of BCL2 and the nuclear factor-kappaB1/MMP-9 signaling pathway. Cancer Sci 105: 265-271, 2014.

26. Radha $\mathrm{G}$ and Raghavan SC: BCL2: A promising cancer therapeutic target. Biochim Biophys Acta Rev Cancer 1868: 309-314, 2017.
27. Martini M, De Santis MC, Braccini L, Gulluni F and Hirsch E: PI3K/AKT signaling pathway and cancer: An updated review. Ann Med 46: 372-383, 2014.

28. Polivka J Jr and Janku F: Molecular targets for cancer therapy in the PI3K/AKT/mTOR pathway. Pharmacol Ther 142: 164-175, 2014.

cc) (i) () This work is licensed under a Creative Commons Attribution-NonCommercial-NoDerivatives 4.0 International (CC BY-NC-ND 4.0) License. 